\title{
OPEN IMAGES OF ORDERABLE SPACES
}

\author{
M. HUSEK AND W. KULPA
}

\begin{abstract}
The class of open continuous images of orderable spaces is characterized, and it is shown that it contains all suborderable spaces. Several results concerning open images of metrizable spaces are generalized to higher cardinals, e.g., every completely linearly uniformizable space is an open continuous image of a Baire space $\left(\kappa^{\lambda}\right)_{\lambda}$.
\end{abstract}

The aim of this paper is to solve a problem of van Wouwe [ $\mathbf{v W}]$, by proving that every suborderable space is an open continuous image of an orderable space. Both authors solved the problem independently using practically the same idea; the first author used a concrete description specialized to suborderable spaces, and the second author used a more abstract technique which allowed him to characterize completely all open images of orderable spaces. In addition, we shall characterize open images of orderable spaces with monotone local bases and of linearly uniformizable spaces (also of complete ones-a generalization of Hausdorff's, Michael's and Ponomarev's results).

The notation and terminology from [E] will be used; we explain those terms used frequently in this paper or not occurring in [E]. The word order means linear order here, so that orderable space is a topological space such that there exists an order on the underlying set with all open intervals forming an open base for the topology. A suborderable space is a topological space which can be embedded into an orderable space (another term is a "generalized orderable space"), which is equivalent to being a $T_{1}$-topological space such that there exists an order on the underlying set with some intervals forming an open base for the topology (see [ᄃ] for this equivalence). A subset $A$ of an ordered space is said to be convex (interval-like in [亡̈]) if it contains with any of its two points $x, y$ an interval containing $x, y$. A topological space is called linearly uniformizable if there is a uniformity inducing the topology and having a monotone base of vicinities of the diagonal with respect to inclusion, or covers with respect to refinement. Such a space is suborderable, and it is orderable provided it is, e.g., dense-in-itself-see $\left[\mathbf{K}_{1}, \mathbf{F K}, \mathbf{H}\right]$. A wider class is the class of caterpillar spaces, where every point has a monotone base of its neighborhoods (this class includes first-countable spaces and suborderable spaces with $\chi(x,] \leftarrow, x])=$ $\chi(x,[x, \rightarrow[)$ whenever $x \in \overline{\leftarrow, x[} \cap \overline{] x, \rightarrow[})$. A still wider class is that of butterfly

Received by the editors November 24, 1981.

1980 Mathematics Subject Classification. Primary 54C10, 54F05.

Key words and phrases. Orderable space, suborderable space, linearly uniformizable space, caterpillar space, butterfly space, open image. 
spaces, where every point $x$ has a base of its neighborhoods of a form $\left\{B_{1} \cup\{x\} \cup\right.$ $\left.B_{2} \mid B_{i} \in h_{i}(x), i=1,2\right\}$, where the $h_{i}(x)$ are ordered families of open sets; this class contains all suborderable spaces and is contained in the class of all spaces $X$ with $\chi(x)=\chi(x) \leqslant|X|$ for all $x \in X$. We may, and shall, always assume that both 解 $(x)$ and $\varphi_{2}(x)$ are nonvoid families of nonvoid sets.

The sum $\Sigma\left\{X_{i} \mid i \in I\right\}$ of sets is the set $\left\{(i, x) \mid i \in I, x \in X_{1}\right\}$. By a sum-order (if all $X_{i}$ and $I$ are ordered) we mean the lexicographical order. Usually one omits the sum-indices $i$ in the sum but must then regard the $X_{i}$ as disjoint.

The class of all butterfly spaces (or caterpillar spaces) is closed under formation of subspaces, sums, and open continuous images, but not under products (e.g. $\left(\left(\omega_{0}+\right.\right.$ 1) $\left.\times\left(\omega_{1}+1\right)\right)$ ) and closed continuous images (e.g., the space of real numbers with the set of integers identified to a point).

The class of all linearly uniformizable spaces is closed under formation of subspaces, but not under sums, products, open or closed continuous images (if we restrict ourselves only to linearly uniformizable spaces with the same character, then that class is closed under sums and finite products).

In the sequel, we shall deal with $T_{1}$-spaces only. It is not too difficult to show that if $f$ is an open continuous map on a butterfly space $X$ onto a space $Y$, then for every $x \in X$ either $f x$ is isolated or $\chi(x)=\chi(f x)$.

THEOREM 1. Every butterfly space is an open continuous image of a zero-dimensional orderable space.

Proof. We may suppose that the investigated space has no isolated points since every butterfly space $P$ is an open continuous image of the butterfly space $P^{\prime}$ which is dense-in-itself. (Let $Q$ stand for the rationals and $P^{\prime}=\{(x, q) \in P \times Q \mid$ either $q=0$ or $x$ is isolated in $P\}$.) The topology of $P^{\prime}$ is the coarsest one making $\operatorname{pr}_{P}$ : $P^{\prime} \rightarrow P$ continuous, and $\mathrm{pr}_{Q}: P^{\prime} \rightarrow Q$ continuous at all points $(x, q), x$ being isolated in $P$ (i.e. the sets $\operatorname{pr}_{P}^{-1}(G), G$ open in $P$, and $(x) \times H, x$ isolated in $P, H$ open in $Q$, form an open base of $P^{\prime}$ ). The map $\operatorname{pr}_{P}$ is open. If $P$ is caterpillar or suborderable then $P^{\prime}$ has the same property (for suborderability use the lexicographical order). Suppose now that a dense-in-itself butterfly space $X$ is given together with infinite monotone collections $\Re_{i}^{\prime}(x), i=1,2, x \in X$, from the definition of butterfly spaces and choose an order on $X$. Let

$$
\mathscr{B}(x)=\left(\mathscr{B}_{1}(x), \supset\right)+(x)+\left(\mathscr{G}_{2}(x), \supset\right), \quad \mathscr{B}=\sum\{\mathscr{B}(x) \mid x \in X\}
$$

(lexicographical sums), and define $L(X)$ to be the following subset of the lexicographical product $(X \times \Re)^{\omega}: z=\left\{\left(x_{n}, B_{n}\right)\right\} \in L(X)$ iff for all $n \in \omega$,

(i) $B_{n} \in \mathscr{B}\left(x_{n}\right)$,

(ii) $x_{n+1} \in B_{n}$,

(iii) $B_{n+1} \subset B_{n}$,

and there is some $n \in \omega$ such that $B_{n}=\left(x_{n}\right)$.

For the above $z \in L(X)$ we let $f z=x_{n}$. Clearly, $f$ maps $L(X)$ onto $X$ and we shall prove that it is continuous and open in the orderable topology on $L(X)$.

Let $x=\left\{\left(x_{n}, X_{n}\right)\right\}$ be a point of $L(X)$ and $A$ a neighborhood of $f x$ in $X$. Let $k$ be the first $n$ with $\left|X_{n}\right|=1$. There are $B^{\prime} \in \mathscr{B}_{1}(f x), B^{\prime \prime} \in \mathscr{\Re}_{2}(f x)$ such that $B^{\prime} \cup B^{\prime \prime} \subset$ $A$, and $B^{\prime} \cup B^{\prime \prime} \subset B_{k-1}$ if $k>0$. If we define $u=\left\{\left(u_{n}, U_{n}\right)\right\}, v=\left\{\left(v_{n}, V_{n}\right)\right\}$ to be 
points of $L(X)$ with the same $n$th coordinates as $x$ has for $n<k$, and $\left(u_{k}, U_{k}\right)=$ $\left(x_{k}, B^{\prime}\right),\left(v_{k}, V_{k}\right)=\left(x_{k}, B^{\prime \prime}\right)$, then $u<x<v$ and $\left.f\right] u, v[\subset A$. Thus $f$ is continuous.

We shall now prove that $f$ is open. Let $u, v, z \in L(X), u<z<v$, where $u=\left\{\left(u_{n}, U_{n}\right)\right\}, v=\left\{\left(v_{n}, V_{n}\right)\right\}, z=\left\{\left(z_{n}, Z_{n}\right)\right\}$. Let $k$ be the last $n$ with $Z_{n}$ open (if $Z_{0}=\left(z_{0}\right)$ we put $\left.k=-1, Z_{-1}=X\right)$. Then there are $B^{\prime} \in h_{1}\left(z_{k+1}\right), B^{\prime \prime} \in Z_{2}\left(z_{k+1}\right)$ such that $B^{\prime} \cup B^{\prime \prime} \subset Z_{k}$ and $B^{\prime}>U_{k+1}$ provided $z_{k+1}=u_{k+1}, B^{\prime \prime}<V_{k+1}$ provided $z_{k+1}=v_{k+1}$. For $x \in B^{\prime} \cup B^{\prime \prime}$ we define $\left(x_{n}, B_{n}\right)=\left(z_{n}, Z_{n}\right)$ for $n \leqslant k, x_{k+1}=$ $z_{k+1}, B_{k+1}=B^{\prime}$ or $B_{k+1}=B^{\prime \prime}$ depending on whether $x \in B^{\prime}$ or $x \in B^{\prime \prime}$, and $\left(x_{n}, B_{n}\right)=(x,(x))$ for $n>k+1$. Clearly, $u<\left\{\left(x_{n}, B_{n}\right)\right\}<v$, so that $\left.f\right] u, v\left[\supset B^{\prime}\right.$ $\cup B^{\prime \prime}$. Consequently, $f$ is open.

The zero-dimensionality of $L(X)$ follows from the fact that for any $z=$ $\left\{\left(z_{n}, Z_{n}\right)\right\} \in L(X)$ with the last open set $Z_{k}$, the sets $\left\{\left\{\left(u_{n}, U_{n}\right)\right\} \mid\left(u_{n}, U_{n}\right)=\right.$ $\left(z_{n}, Z_{n}\right)$ for $\left.n \leqslant k, u_{k+1}=z_{k+1}, U_{k+1}=B\right\}$, where $B \in \mathscr{B}\left(z_{k+1}\right)$, form clopen basic neighborhoods of $z$ in $L(X)$.

COROLlary 1. The class of continuous open images of (zero-dimensional) orderable spaces coincides with the class of all butterfly spaces.

Remarks. (a) The correspondence $\left\{P \rightarrow P^{\prime}\right\},\left\{f \rightarrow\left(f \times 1_{Q}\right) / P\right\}$ is an idempotent functor $L_{0}$ on the category of all topological spaces with open continuous maps onto its subcategory of all dense-in-itself spaces. The projections $\left\{P^{\prime} \rightarrow P\right\}$ form a natural transformation from $L_{0}$ to $1_{\text {Top }}$. The functor $L_{0}$ preserves many nice properties. It may be regarded as a weak coreflection.

(b) If the space $X$ is caterpillar, then the constructed space $L(X)$ is caterpillar as well so that we have another corollary (also using the fact mentioned in the introduction that an open continuous image of a caterpillar space is caterpillar, too).

COROLlary 2. The class of all open continuous images of (zero-dimensional) orderable caterpillar spaces coincides with the class of all caterpillar spaces.

(c) If $X$ is a first countable space, then $L(X)$ is metrizable (if $\mathscr{T}_{1}(x)=\mathscr{T}_{2}(x)=$ $\left\{B_{n}(x) \mid n \in \omega\right\}$ is a base at $x$, then

$$
\begin{aligned}
U_{k}=\left\{\left(\left\{u_{n}, U_{n}\right\},\left\{v_{n}, V_{n}\right\}\right) \mid\right. & \exists n_{0}, x: u_{i}=v_{i}, U_{i}=V_{i} \\
& \text { if } \left.i<n_{0}, u_{n_{0}}=v_{n_{0}}, U_{n_{0}} \cup V_{n_{0}} \subset B_{k}(x)\right\}, \quad k \in \omega,
\end{aligned}
$$

are equivalences which induce, as a base for a uniformity, the topology of $L(X)$ ). Consequently, $L(X)$ may be embedded into a Baire space. Thus we have got Ponomarev's result $[\mathbf{P}]$ :

COROLlaRY 3. Every first countable space is an open continuous image of a subspace of a Baire space.

Of course, the direct proof is simpler than using our Theorem 1, but one can guess from the above consideration how to generalize the Ponomarev theorem to higher cardinals. To do that, we prefer another approach which is closer to the original one in $[\mathbf{P}]$.

At the beginning we shall proceed as Ponomarev did. Let $\mathscr{B}$ be an open base for $X$ containing $X$, where $X$ is a caterpillar space. Denote by $L_{1}(X, \Re)$ the following 
subset of $9_{\mathcal{B}}{ }^{X}: u=\left\{u_{\xi} \mid \xi \in \chi X\right\}$ belongs to $L_{1}\left(X, \mathscr{S}_{3}\right)$ if $\left\{u_{\xi}\right\}$ is a base at some point $f u$ of $X$ and there is an ordinal $p u$ such that $u_{\beta} \subset u_{\alpha} \neq X$ for $\alpha<\beta<p u$ and $u_{\alpha}=X$ for $\alpha \geqslant p u$ (then, of course, $p u=\sup \left\{\alpha \mid\right.$ Int $\left.\cap\left\{u_{\xi} \mid \xi \in \alpha\right\}\right\} \neq \varnothing$ ). If we regard $\mathscr{S}$ as a discrete space, $L_{1}\left(X, \mathscr{S}^{\prime}\right)$ the subspace of the product space $\varphi^{X}{ }^{X}$, then $f: L_{1}(X, \mathscr{B}) \rightarrow X$ is a continuous surjection but it need not be open (it is open provided $\chi(x)=\chi X$ for all nonisolated $x \in X$, which is the case of first countable spaces or of linearly uniformizable spaces).

If we ignore the coordinates $u_{\xi}$ for $\xi \geqslant p u$ we obtain the set $L_{2}(X, 1$,$) endowed$ with the topology having

$$
\left\{\left\{v_{\xi}\right\} \in L_{1}(X, \Re) \mid v_{\xi}=u_{\xi} \text { for } \xi \in K\right\}, \quad K \subset \chi X,|K|<\omega, \sup K<p u,
$$

as a local base at $\left\{u_{\xi}\right\}$. Then $f: L_{2}(X, \mathscr{Q}) \rightarrow X$ is an open continuous surjection. It is easy to see that one can modify the topology of $L_{2}(X, 9)$ to obtain another space $L_{3}(X, \mathscr{G})$ such that $f: L_{3}(X, \mathscr{G}) \rightarrow X$ is open and continuous, and $L_{3}(X, \mathscr{H})$ is caterpillar: one omits $|K|<\omega$ in the above definition of the topology. (The spaces $L_{1}, L_{2}$ need not be caterpillar if $\chi X$ is uncountable.) The space $L_{3}$ is nonarchimedean (the defined base with $K$ initial segments has the property that any two of its members are either disjoint or comparable by inclusion), thus by $\left[\mathbf{K}_{2}\right], L_{3}\left(X, \mathscr{O}^{7}\right)$ is orderable provided it is dense-in-itself (in general it is suborderable). This gives another proof of Corollary 2.

Since open continuous images of caterpiller spaces preserve characters, one cannot expect an open continuous preimage of a general caterpillar space to be linearly uniformizable. But if $\chi(x)=\chi X$ for every nonisolated $x \in X$, then $L_{3}(X$, 伯) is linearly uniformizable: $U_{\alpha}=\left\{\left(\left\{u_{\xi}\right\},\left\{v_{\xi}\right\}\right) \mid u_{\xi}=v_{\xi}\right.$ for $\left.\xi \in \alpha\right\}, \alpha \in \chi X$, form a base for such a uniformity. Thus we have the following generalization of the Ponomarev theorem (every linearly uniformizable zero-dimensional space can be embedded into a generalized Baire space (see Remark (g) for the definition)):

THEOREM 2. The class of all open continuous images of linearly uniformizable orderable spaces $X$ with $\chi X=\kappa$ coincides with the class of all caterpillar spaces $X$ having the property $\chi(x)=\kappa$ for every nonisolated $x \in X$.

REMARKS. (d) Sometimes it may be better to look at points of $L_{3}(X, 93)$ as families $\left\{u_{\xi} \mid \xi \in \chi(x)\right\}$, where $\left\{u_{\xi}\right\} \subset \Re$ is a local base at $x$ such that $\alpha \in \beta \in \chi(x)$ implies $u_{\alpha} \supset u_{\beta}$.

(e) If we take $\Re$ to be all open subsets of $X$, we denote $L_{3}(X, 9)$ by $L_{3}(X)$. To every open continuous map $f: X \rightarrow Y$ ( $X, Y$ caterpillar spaces) one can assign an open continuous map $L_{3}(f)=\left\{\left\{u_{\xi}\right\} \rightarrow\left\{f u_{\xi}\right\}\right\}: L_{3}(X) \rightarrow L_{3}(Y)$. Thus $L_{3}$ is a functor on the category $C$ of all caterpillar spaces with open continuous maps into its subcategory of all zero-dimensional caterpillar orderable spaces. The maps $f: L_{3}(X)$ $\rightarrow X$ form a natural transformation of $L_{3}$ into $1_{\mathcal{C}}$.

(f) Results from [vW] dealing with comparing various classes of open continuous images of suborderable spaces can be proved now in a simpler way by using our Theorem 1. For instance if $X$ is a stratifiable open continuous image of a suborderable space, then $X$ is a butterfly space with countable pseudocharacter, thus a first countable space - consequently, it is a Nagata space. 
(g) The classical Baire space is topologically a power $D^{\omega}$, where $D$ is a discrete space-we shall write it in the form $\lambda^{\omega}$, where $\lambda=|D|$. To generalize such spaces one must take the power $\lambda^{\kappa}, \kappa$ a regular infinite cardinal, with the $\kappa$-box topology. We shall denote that by $\left(\lambda^{\kappa}\right)_{\kappa}$ in agreement with [CN]. Every linearly uniformizable space $X$ can be embedded into $\left(\lambda^{\kappa}\right)_{\kappa}$ (with $\left.\lambda=w X, \kappa=\chi X\right)$. Using generalized Baire spaces, we can prove the following generalization of Hausdorff's result from [Ha] (for complete metric spaces).

THEOREM 3. Every completely linearly uniformizable space $X$ is an open continuous image of the generalized Baire space $\left(w X^{\chi X}\right)_{\chi X}$.

Proof. Let $w X=\lambda, \chi X=\kappa$ and let $u$ be a complete uniformity on $X$ having a monotone base of open covers $\left\{\mathcal{Q}_{\alpha} \mid \alpha \in \kappa\right\}$ with $\vartheta_{0}=(X)$ and such that

$$
\wedge\left\{\mathcal{Q u}_{\beta} \mid \mathscr{G}_{\mathcal{B}} \in \alpha\right\}=\mathcal{Q}_{\alpha} \text { for limit } \alpha
$$

if $\kappa>\omega$ we may suppose that $\mathcal{Q}_{\alpha}$ are decompositions, for $\kappa=\omega$ let $\mathcal{Q l}_{\alpha}, \alpha>0$, consist of all open balls from a base of $X$ of cardinality $\lambda$ of diameter at most $2^{-\alpha}$ for a given metric. We may also assume that for $U \in \mathcal{Q}_{\alpha}$ the set $\left\{V \in \mathcal{Q}_{\alpha+1} \mid V \subset U\right\}$ is well-ordered and forms an initial part of $\lambda$ of length $\varphi U=\left|\left\{V \in \mathcal{Q}_{\alpha+1} \mid V \subset U\right\}\right|$. If $x=\left\{\xi_{\alpha} \mid \alpha \in \kappa\right\} \in \lambda^{\kappa}$, then $f x$ will be the unique point of $\cap\left\{U_{\alpha} \mid \alpha \in \kappa\right\}$, where $U_{\alpha} \in \mathcal{Q}_{\alpha}$ form a chain constructed transfinitely in the following way: suppose that all $U_{\beta}$ are defined for $\beta \in \alpha$ (clearly, $U_{0}=X$ ); if $\alpha$ is limit, then $U_{\alpha}=\bigcap_{\beta \in \alpha} U_{\beta}$, if $\alpha=\beta+1$, then $U_{\alpha}$ is the $\eta$ th member of $\left\{V \in \mathcal{Q}_{\alpha} \mid V \subset U_{\beta}\right\}$, where $\xi_{\alpha}=\xi \cdot \varphi U+\eta$. The map $f$ is onto and is continuous and open because it maps basic open sets $\left\{\left\{\xi_{\alpha}\right\} \in \lambda^{\kappa} \mid \xi_{\alpha}=\eta_{\alpha}\right.$ for $\left.\alpha \leqslant \beta\right\}, \beta \in \kappa,\left\{\eta_{\alpha}\right\} \in \lambda^{\beta}$, onto the members of ฯl $_{\beta}, \beta \in \kappa$.

\section{REFĖRENCES}

[CN] W. W. Comfort and S. Negrepontis, The theory of ultrafilters, Springer-Verlag, Berlin, 1974.

[ЦC] E. Čech, Topological spaces (rev. ed. by Z. Frolik and M Katětov), Academia. Prague, 1966.

[E] R. Engelking, General topologi, PWN, Warsaw, 1977.

[FK] R. Frankiewicz and W. Kulpa, On order topology of spaces having uniform linearly ordered bases, Comment. Math. Univ. Carolin. 20 (1979), 37-41.

[Ha] F. Hausdorf, Über innere Abbildungen, Fund. Math. 23 (1934), 279-291.

[H] M. Hušek, Linearly uniformizable spaces, Rapport Wisk. Sem. Vrije Univ. 119 (1980), 19 pp.

$\left[\mathbf{K}_{\mathbf{1}}\right]$ D. Kurepa, On the existence of pseudometric nontotally orderable spaces, Glasnik Mat.-Fiz. Astronom. Ser. II Društvo Mat. Fiz. Hrvatske 18 (1963), 183-194.

$\left[\mathbf{K}_{\mathbf{2}}\right]$, Sur l'escart abstrait, Glasnik Mat.-Fiz. Astronom. Ser. II Društvo Mat. Fiz. Hrvatske 11 (1956), 105-134.

[Mi] E. Michael, A theorem on semi-continuous set-valued functions, Duke Math. J. 26 (1959), 647-651.

[P] V. I. Ponomarev, Axioms of countability and continuous mappings, Bull. Acad. Polon. Sci. Sér. Math. Astronom. Phys. 8 (1960), 127-133.

[vW] J. van Wouwe, GO-spaces and generalizations of metrizability, Math. Centre Tract 104, Math. Centrum, Amsterdam, 1979.

Matematicky Ústav Karlovy University, Sokolovská 83, 18600 Praha, Czechoslovakia

Instytut Matematyki Uniwersytet Sląski, Bankowa 14, 40-007 Katowice, Poland 